\title{
Bounded Solution of Cauchy Type Singular Integral Equation of The First Kind Using Differential Transform Method
}

\author{
M. Abdulkawi ${ }^{1}$, S. A. H. Moayad ${ }^{2}$ \\ ${ }^{1,2}$ Department of Mathematics, Faculty of Science and Arts, \\ Najran University, Saudi Arabia \\ ${ }^{1}$ Email: kawi2011@gmail.com
}

\section{ABSTRACT}

In this paper, an efficient approximate solution for solving the Cauchy type singular integral equation of the first kind is presented. Bounded solution of the Cauchy type singular Integral equation is discussed. Two types of kernel, separable and convolution, are considered. The differential transform method is used in the solution. New theorems for transformation of Cauchy singular integrals are given with proofs. Numerical results are shown to illustrate the efficiency and accuracy of the approximate solution.

2000 MSC: 65R20, 45E05, 45E10

Keywords: Integral Equation, Cauchy Kernel, Convolution Kernel, Degenerate Kernel, Differential Transform Method.

Language: English

Date of Submission: 2018-01-27

Date of Acceptance: 2018-03-03

Date of Publication: 2018-04-30

ISSN: 2347-1921

Volume: 14 Issue: 01

Journal: Journal of Advances in Mathematics

Website: https://cirworld.com

This work is licensed under a Creative Commons Attribution 4.0 International License. 


\section{INTRODUCTION}

The Cauchy type singular integral equation (CSIE)

$$
\int_{-1}^{1} \frac{\varphi(t)}{t-x} d t+\int_{-1}^{1} k(x, t) \varphi(t) d t=f(x), \quad-1<x<1
$$

where $K(x, t)$ and $f(x)$ are given real valued functions belonging to the Holder class and $\varphi(t)$ is to be determined, occurs in varieties of mixed boundary value problems of mathematical physics, isotropic elastic bodies involving cracks and other related problems [1-3]. The singular integral in CSIE (1) is considered as Cauchy principal value integral. Dezhborda et al. [5] investigated the numerical solution of various cases of CSIE (1) using reproducing kernel Hilbert space (RKHS) method. Chakrabarti and Berge [6] have proposed an approximate method to solve CSIE (1) using polynomial approximation of degree $n$ and collocation points chosen to be the zeros of Chebyshev polynomial of the first kind for all cases. They showed that the approximate method is exact when the force function $f(t)$ is linear. Abdulkawi [1] discussed the numerical solution of CSIE (1) for tow cases, unbounded and bounded. He approximated the unknown function by weighted Chebyshev polynomials of the first and second kind, respectively, and used Lagrange-Chebyshev interpolation to approximate the regular kernel. Eshkuvatov et al. [7] discussed approximate solution of CSIE (1) when $K(x, t)=0$ for four cases. They used weighted Chebyshev polynomials of the first, second, third and fourth kinds. They showed that the numerical solution is identical with the exact solution when the force function is a polynomial of degree one. Abdulkawi and Akran [2] discussed the numerical solution of CSIE (1) when $K(x, t)=0$ for bounded case. They used the Differential Transform Method in the solution. Dardery and Allan [10] discussed approximate solution of CSIE (1) by means of Chebyshev polynomials of first, second, third and fourth kind to obtain systems of linear algebraic equations which are solved numerically.

In this paper, we focus our discussion on approximate solution of equation

$$
\int_{-1}^{1} \frac{g(t)}{t-x} d t+\int_{-1}^{1} k(x, t) g(t) d t=f(x), \quad-1<x<1
$$

for two types of the kernel, Separable and Convolution.

It is known that the analytical solution of the equation (2) for bounded case is given by the following expression [11].

$$
\left.\begin{array}{l}
g(x)+\int_{-1}^{1} N_{1}(x, \tau) g(\tau) d \tau=F_{1}(x), \\
N_{1}(x, \tau)=-\frac{\sqrt{1-x^{2}}}{\pi^{2}} \int_{-1}^{1} \frac{k(t, \tau)}{\sqrt{1-t^{2}}(t-x)} d t, \\
F_{1}(x)=-\frac{\sqrt{1-x^{2}}}{\pi^{2}} \int_{-1}^{1} \frac{f(t)}{\sqrt{1-t^{2}}(t-x)} d t .
\end{array}\right\}
$$

where

$$
\int_{-1}^{1} g(t) d t=0
$$




\section{DIFFERENTIAL TRANSFORM METHOD}

The transformation of the kth derivative of a function in one variable is as follows:

$$
F(k)=\frac{1}{k !}\left[\frac{d^{k}}{d x^{k}} f(x)\right]_{x=x_{0}}
$$

and the inverse transformation is defined by

$$
f(x)=\sum_{k=0}^{\infty} F(k)\left(x-x_{0}\right)^{k} .
$$

The following theorems can be deduced from Eqs. (5) and (6) [4].

Theorem 1. if $f(x)=g(x) \pm h(x)$, then

$$
F(k)=G(k) \pm H(k) .
$$

Theorem 2. if $f(x)=c g(x)$, then

$$
F(k)=c G(k)
$$

Where $c$ is constant.

Theorem 3. if $f(x)=\frac{d^{n} g(x)}{d x^{n}}$, then

$$
F(k)=\frac{(k+n) !}{k !} G(k+n) .
$$

Theorem 4. if $f(x)=g(x) h(x)$, then

$$
F(k)=\sum_{k_{1}=0}^{k} G\left(k_{1}\right) H\left(k-k_{1}\right) .
$$

Theorem 5. if $f(x)=x^{n}$, then $F(k)=\delta(k-n)$, where

$$
\delta(k-n)=\left\{\begin{array}{l}
1, k=n, \\
0, k \neq n .
\end{array}\right.
$$

The following Lemma and Theorems are used in this paper [2].

Lemma 1. If $C(k)=\int_{-1}^{1} \frac{t^{k}}{\sqrt{1-t^{2}}} d t$ then 


$$
C(k)=\left\{\begin{array}{lll}
\pi & \text { if } & k=0, \\
0 & \text { if } & k \text { is odd }, \\
\frac{\pi}{2^{k}}\left(\frac{k}{2}\right) & \text { if } & k \text { is even } .
\end{array}\right.
$$

Theorem 6. If $g(x)=\int_{-1}^{1} \frac{\sqrt{1-t^{2}} \varphi(t)}{t-x} d t$, then the differential transform of $g(x)$ is

$$
G(m)=\sum_{k=1}^{N} \Phi(k) \sum_{r=1}^{k-1} \delta(m-r)(C(k-r-1)-C(k-r+1))-\pi \sum_{k_{1}=0}^{m} \delta\left(k_{1}-1\right) \Phi\left(m-k_{1}\right)
$$

where $\Phi(k)$ is the differential transform of $\varphi(t)$ and $C(k)$ is defined by Lemma 1 .

\section{THE SCHEME OF THE APPROXIMATE SOLUTION}

The approximate solution is derived using the following procedures

1- The unknown function $g(t)$ is approximated as:

$$
g(t)=\sqrt{1-t^{2}} \varphi(t)
$$

where is regular function, so Eq.(1) becomes

$$
\int_{-1}^{1} \frac{\sqrt{1-t^{2}} \varphi(t)}{(t-x)} d t+\int_{-1}^{1} \sqrt{1-t^{2}} k(x, t) \varphi(t) d t=f(x), \quad-1<x<1
$$

2- Two Theorems for evaluating the following singular integral for two types of kernels are proved

$$
\int_{-1}^{1} \sqrt{1-t^{2}} k(x, t) \varphi(t) d t
$$

3- Lemma 1 is used for evaluating the singular integral

$$
\int_{-1}^{1} \frac{t^{k}}{\sqrt{1-t^{2}}} d t, \quad \forall k \in Z^{+}
$$

4- The following condition is imposed to obtain the unique solution

$$
\int_{-1}^{1} g(t) d t=0 .
$$


5- Maple codes are developed and run on the personal computer with Pentium 4 processors to obtain the numerical results.

Theorem 7. If

$$
g(x)=\int_{-1}^{1} \sqrt{1-t^{2}} k(x, t) \varphi(t) d t
$$

where $k(x, t)=a(x) b(t)$, then the differential transform of $\mathrm{g}(\mathrm{x})$ is

$$
G(m)=\left(\sum_{k_{1}=0}^{N} \sum_{k_{2}=0}^{N} \Phi\left(k_{1}\right) B\left(k_{2}\right)\left(C\left(k_{1}+k_{2}\right)-C\left(k_{1}+k_{2}+2\right)\right)\right) A(m), \quad N \rightarrow \infty
$$

where $\Phi\left(k_{1}\right), B\left(k_{2}\right), A(m)$ are the differential transform of $\varphi(x), b(t), a(x)$ respectively.

\section{Proof :}

Let

$$
\begin{gathered}
g(x)=\int_{-1}^{1} \sqrt{1-t^{2}} a(x) b(t) \varphi(t) d t \\
=a(x) \int_{-1}^{1} \sqrt{1-t^{2}} b(t) \varphi(t) d t
\end{gathered}
$$

Using Maclaurin series of the functions $\varphi(t)$ and $b(t)$ into (13) yields

$$
\begin{aligned}
g(x) & =a(x) \int_{-1}^{1} \sqrt{1-t^{2}} \sum_{k_{1}=0}^{\infty} \Phi\left(k_{1}\right) t^{k_{1}} \sum_{k_{2}=0}^{\infty} \mathrm{B}\left(k_{2}\right) t^{k_{2}} d t \\
& =a(x) \sum_{k_{1}=0}^{\infty} \sum_{k_{2}=0}^{\infty} \Phi\left(k_{1}\right) \mathrm{B}\left(k_{2}\right) \int_{-1}^{1} \sqrt{1-t^{2}} t^{k_{1}+k_{2}} d t
\end{aligned}
$$

From (14) we obtain

$$
\begin{aligned}
g(x) & =a(x) \sum_{k_{1}=0}^{\infty} \sum_{k_{2}=0}^{\infty} \Phi\left(k_{1}\right) \mathrm{B}\left(k_{2}\right) \int_{-1}^{1} \frac{\left(1-t^{2}\right) t^{k_{1}+k_{2}}}{\sqrt{1-t^{2}}} d t \\
& =a(x) \sum_{k_{1}=0}^{\infty} \sum_{k_{2}=0}^{\infty} \Phi\left(k_{1}\right) \mathrm{B}\left(k_{2}\right) \int_{-1}^{1} \frac{t^{k_{1}+k_{2}}-t^{k_{1}+k_{2}+2}}{\sqrt{1-t^{2}}} d t \\
& =a(x) \sum_{k_{1}=0}^{\infty} \sum_{k_{2}=0}^{\infty} \Phi\left(k_{1}\right) \mathrm{B}\left(k_{2}\right)\left(\int_{-1}^{1} \frac{t^{k_{1}+k_{2}}}{\sqrt{1-t^{2}}} d t-\int_{-1}^{1} \frac{t^{k_{1}+k_{2}+2}}{\sqrt{1-t^{2}}} d t\right)
\end{aligned}
$$


Due to Lemma 1 , we have

$$
g(x)=a(x) \sum_{k_{1}=0}^{\infty} \sum_{k_{2}=0}^{\infty} \Phi\left(k_{1}\right) \mathrm{B}\left(k_{2}\right)\left(C\left(k_{1}+k_{2}\right)-C\left(k_{1}+k_{2}+2\right)\right)
$$

Using Theorem 2 and 6 into (16) we obtain

$$
G(m)=\left(\sum_{k_{1}=0}^{N} \sum_{k_{2}=0}^{N} \Phi\left(k_{1}\right) \mathrm{B}\left(k_{2}\right)\left(C\left(k_{1}+k_{2}\right)-C\left(k_{1}+k_{2}+2\right)\right)\right) \mathrm{A}(m), \quad N \rightarrow \infty
$$

The proof is complete.

Theorem 8. If

$$
g(x)=\int_{-1}^{1} \sqrt{1-t^{2}} k(x, t) \varphi(t) d t
$$

where $k(x, t)=f_{1}(x-t)$, then the differential transform of $g(x)$ is

$$
\begin{aligned}
G(m) & =\sum_{k_{1}=0}^{N} \Phi\left(k_{1}\right) \sum_{k_{2}=0}^{N} F_{1}\left(k_{2}\right) \sum_{k=0}^{k_{2}}(-1)^{k}\left(\begin{array}{l}
k_{2} \\
k
\end{array}\right) \delta\left(k_{2}-k-m\right)\left(C\left(k+k_{1}\right)-C\left(k+k_{1}+2\right)\right) \\
, N \rightarrow \infty &
\end{aligned}
$$

where $\Phi\left(k_{1}\right), F_{1}\left(k_{2}\right)$ are the differential transform of $\varphi(x), f_{1}(x-t)$ respectively .

\section{Proof :}

Let

$$
g(x)=\int_{-1}^{1} \sqrt{1-t^{2}} f_{1}(x-t) \varphi(t) d t
$$

Using Maclaurin series of the function $\varphi(t)$ and $f_{1}(x-t)$ into (19) yields

$$
\begin{aligned}
g(x) & =\int_{-1}^{1} \sqrt{1-t^{2}} \sum_{k_{1}=0}^{\infty} \Phi\left(k_{1}\right) t^{k_{1}} \sum_{k_{2}=0}^{\infty} F_{1}\left(k_{2}\right)(x-t)^{k_{2}} d t \\
& =\sum_{k_{1}=0}^{\infty} \sum_{k_{2}=0}^{\infty} \Phi\left(k_{1}\right) F_{1}\left(k_{2}\right) \int_{-1}^{1} \sqrt{1-t^{2}} t^{k_{1}}(x-t)^{k_{2}} d t
\end{aligned}
$$

From (20) we obtain

$$
g(x)=\sum_{k_{1}=0}^{\infty} \sum_{k_{2}=0}^{\infty} \Phi\left(k_{1}\right) F_{1}\left(k_{2}\right) \int_{-1}^{1} \frac{\left(1-t^{2}\right) t^{k_{1}}(x-t)^{k_{2}}}{\sqrt{1-t^{2}}} d t
$$


By using Binomial theorem for function $(x-t)^{k_{2}}$ into (21) yields

$$
\begin{aligned}
g(x) & =\sum_{k_{1}=0}^{\infty} \sum_{k_{2}=0}^{\infty} \Phi\left(k_{1}\right) F_{1}\left(k_{2}\right) \int_{-1}^{1} \frac{\left(1-t^{2}\right) t^{k_{1}} \sum_{k=0}^{k_{2}}(-1)^{k}\left(\begin{array}{l}
k_{2} \\
k
\end{array}\right) t^{k} x^{k_{2}-k}}{\sqrt{1-t^{2}}} d t \\
& =\sum_{k_{1}=0}^{\infty} \sum_{k_{2}=0}^{\infty} \Phi\left(k_{1}\right) F_{1}\left(k_{2}\right) \sum_{k=0}^{k_{2}}(-1)^{k}\left(\begin{array}{l}
k_{2} \\
k
\end{array}\right) x^{k_{2}-k}\left(\int_{-1}^{1} \frac{\left(1-t^{2}\right) t^{k+k_{1}}}{\sqrt{1-t^{2}}} d t\right) \\
& =\sum_{k_{1}=0}^{\infty} \sum_{k_{2}=0}^{\infty} \Phi\left(k_{1}\right) F_{1}\left(k_{2}\right) \sum_{k=0}^{k_{2}}(-1)^{k}\left(\begin{array}{l}
k_{2} \\
k
\end{array}\right) x^{k_{2}-k}\left(\int_{-1}^{1} \frac{t^{k+k_{1}}-t^{k+k_{1}+2}}{\sqrt{1-t^{2}}} d t\right)
\end{aligned}
$$

Due to Lemma 1 we have

$$
G(m)=\sum_{k_{1}=0}^{N} \Phi\left(k_{1}\right) \sum_{k_{2}=0}^{N} F_{1}\left(k_{2}\right) \sum_{k=0}^{k_{2}}(-1)^{k}\left(\begin{array}{l}
k_{2} \\
k
\end{array}\right) x^{k_{2}-k}\left(C\left(k+k_{1}\right)-C\left(k+k_{1}+2\right)\right)
$$

Using Theorem 2 and 5 into (23) we obtain

$$
\begin{gathered}
G(m)=\sum_{k_{1}=0}^{N} \Phi\left(k_{1}\right) \sum_{k_{2}=0}^{N} F_{1}\left(k_{2}\right) \sum_{k=0}^{k_{2}}(-1)^{k}\left(\begin{array}{l}
k_{2} \\
k
\end{array}\right) \delta\left(k_{2}-k-m\right)\left(C\left(k+k_{1}\right)-C\left(k+k_{1}+2\right)\right) \\
, N \rightarrow \infty
\end{gathered}
$$

The proof is complete.

\section{SOLUTION OF THE SYSTEM FOR SEPARABLE KERNEL}

Taking the differential transform for two sides of Eq.(7) and using Theorems 6 and 7 yields

$$
\left.\begin{array}{l}
\sum_{k=1}^{N} \Phi(k) \sum_{k_{1}=0}^{k-1} \delta\left(m-k_{1}\right)\left[C\left(k-k_{1}-1\right)-C\left(k-k_{1}+1\right)\right]- \\
\quad-\pi \sum_{k_{2}=0}^{m} \delta\left(k_{2}-1\right) \Phi\left(m-k_{2}\right)+ \\
+\left[\sum_{k_{1}=0}^{N} \Phi\left(k_{1}\right) \sum_{k_{2}=0}^{N} G_{2}\left(k_{2}\right)\left[C\left(k_{1}+k_{2}\right)-C\left(k_{1}+k_{2}+2\right)\right]\right] G_{1}(m)=F(m), \\
\quad, N \rightarrow \infty, \quad m=1,2, \ldots, N+1 .
\end{array}\right\}
$$

By solving the system (24) we obtain the unknown coefficients $\{\Phi(k)\}_{0}^{N}$. 
Therefore, the approximate solution of Eq. (7) will be of the form

$$
\varphi_{n}(x) \square \sum_{k=0}^{N} \Phi(k) x^{k}
$$

and then the solution of Eq. (1) will be of the form

$$
g(x) \square \sqrt{1-x^{2}} \sum_{k=0}^{N} \Phi(k) x^{k} .
$$

\section{SOLUTION OF THE SYSTEM FOR CONVOLUTION KERNEL}

Taking the differential transform for two sides of Eq.(7) and using Theorems 9 and 6 yields

$$
\begin{aligned}
& \sum_{k=1}^{N} \Phi(k) \sum_{k_{1}=0}^{k-1} \delta\left(m-k_{1}\right)\left[C\left(k-k_{1}-1\right)-C\left(k-k_{1}+1\right)\right]- \\
& \quad-\pi \sum_{k_{2}=0}^{m} \delta\left(k_{2}-1\right) \Phi\left(m-k_{2}\right) \\
& \left.+\sum_{k_{1}=0}^{N} \Phi\left(k_{1}\right) \sum_{k_{2}=0}^{N} F_{1}\left(k_{2}\right) \sum_{k=0}^{k_{2}}(-1)^{k}\left(\begin{array}{l}
k_{2} \\
k
\end{array}\right) \delta\left(k_{2}-k-m\right)\left(C\left(k+k_{1}\right)-C\left(k+k_{1}+2\right)\right)\right\} \\
& =F(m), \quad N \rightarrow \infty, \quad m=1,2, \ldots, N+1 .
\end{aligned}
$$

By solving the system (25) we obtain the unknown coefficients $\{\Phi(k)\}_{0}^{N}$.

Therefore, the approximate solution of Eq. (7) will be of the form

$$
\varphi_{n}(x) \square \sum_{k=0}^{N} \Phi(k) x^{k}
$$

and then the solution of Eq. (1) will be of the form

$$
g(x) \square \sqrt{1-x^{2}} \sum_{k=0}^{N} \Phi(k) x^{k} .
$$

\section{NUMERICAL RESULTS}

The errors of the approximate solutions in the following examples are computed as the absolute value of the difference between the exact and approximate solutions.

Example 1. Consider the following integral equation

$$
\int_{-1}^{1} \frac{g(t)}{t-x} d t+\int_{-1}^{1} t \sin x g(t) d t=-\pi x^{3}, \quad-1<x<1
$$


It is not difficult to verify that the exact solution of Eq.(26) is

$$
g(t)=\sqrt{1-t^{2}}\left(t^{2}+\frac{1}{2}\right) .
$$

It is clear that the differential Transform of $f(x)=-\pi x^{3}$ is

$$
\left.\begin{array}{l}
F(1)=0, \\
F(2)=0, \\
F(3)=-\pi, \\
F(k)=0, k=4,5, \ldots
\end{array}\right\}
$$

It is clear that $k(x, t)=t \sin x$, which is Separable Kernel .

Let $g_{1}(x)=\sin x, g_{2}(t)=t$, then the differential transform of $g_{1}$ and $g_{2}$ respectively are

$$
\begin{aligned}
& G_{1}(k)= \begin{cases}\frac{(-1)^{\frac{k-1}{2}}}{k !}, & k=1,3, \ldots \\
0, & k=0,2, \ldots\end{cases} \\
& \left.G_{2}(1)=1, \quad G_{2}(2)=0, \quad G_{2}(3)=0 .\right\}
\end{aligned}
$$

Using the system (24) with $N=2$ we have

$$
\begin{aligned}
& \sum_{k=1}^{2} \Phi(k) \sum_{k_{1}=0}^{k-1} \delta\left(m-k_{1}\right)\left[C\left(k-k_{1}-1\right)-C\left(k-k_{1}+1\right)\right]- \\
& \quad-\pi \sum_{k_{2}=0}^{m} \delta\left(k_{2}-1\right) \Phi\left(m-k_{2}\right)+ \\
& +\left[\sum_{k_{1}=0}^{2} \Phi\left(k_{1}\right) \sum_{k_{2}=0}^{2} G_{2}\left(k_{2}\right)\left[C\left(k_{1}+k_{2}\right)-C\left(k_{1}+k_{2}+2\right)\right]\right] G_{1}(m)=F(m), \\
& \quad m=1,2,3 .
\end{aligned}
$$

Which equivalent to the system 


$$
\left.\begin{array}{l}
\Phi(0)-\frac{1}{8} \Phi(1)-\frac{1}{2} \Phi(2)=0 \\
\Phi(1)=0 \\
\frac{1}{48} \Phi(1)+\Phi(2)=1
\end{array}\right\}
$$

Solving the system (31) yields

$$
\left.\Phi(0)=\frac{1}{2}, \quad \Phi(1)=0, \quad \Phi(2)=1\right\}
$$

Thus the approximate solution of Eq.(26) is

$$
g(t)=\sqrt{1-t^{2}}\left(\frac{1}{2}+t^{2}\right)
$$

which is identical to the exact solution.

Example 2. Consider the following integral equation

$$
\int_{-1}^{1} \frac{g(t)}{t-x} d t+\int_{-1}^{1}\left(x^{3}+x t^{2}\right) g(t) d t=x^{3}+x, \quad-1<x<1
$$

It is not difficult to verify that the exact solutions of Eq.(33) is

$$
g(x)=\sqrt{1-x^{2}}\left(\frac{-92}{31 \pi}-\frac{88}{31 \pi} x^{2}\right) .
$$

It is clear that the differential Transform of $f(x)=x^{3}+x$ is

$$
\left.\begin{array}{l}
F(0)=0, \\
F(1)=1, \\
F(2)=0, \\
F(3)=1, \\
F(k)=0, k=4, \ldots
\end{array}\right\}
$$

It is clear that $k(x, t)=x^{3}+x t^{2}$, which is separable kernel.

Let $g_{1}(x)=x^{3}, g_{2}(x)=1, g_{1.1}(x)=x, g_{2.2}(x)=t^{2}$, then the differential transform of $g_{1}, g_{2}, g_{1.1}$ and $g_{2.2}$ respectively are 


$$
\left.\begin{array}{l}
G_{1}(0)=0, G_{1}(1)=0, G_{1}(2)=0, G_{1}(3)=1, \\
G_{2}(0)=1, G_{2}(1)=0, G_{2}(2)=0, G_{2}(3)=0, \\
G_{1.1}(0)=0, G_{1.1}(1)=1, G_{1.1}(2)=0, G_{1.1}(3)=0, \\
G_{2.2}(0)=0, G_{2.2}(1)=0, G_{2.2}(2)=1, G_{2.2}(3)=0 .
\end{array}\right\}
$$

Using the system (24) with $N=2$, we have

$$
\begin{aligned}
& \sum_{k=1}^{2} \Phi(k) \sum_{k_{1}=0}^{k-1} \delta\left(m-k_{1}\right)\left[C\left(k-k_{1}-1\right)-C\left(k-k_{1}+1\right)\right]- \\
& \quad-\pi \sum_{k_{2}=0}^{m} \delta\left(k_{2}-1\right) \Phi\left(m-k_{2}\right)+ \\
& \quad+\left[\sum_{k_{1}=0}^{2} \Phi\left(k_{1}\right) \sum_{k_{2}=0}^{2} G_{2}\left(k_{2}\right)\left[C\left(k_{1}+k_{2}\right)-C\left(k_{1}+k_{2}+2\right)\right]\right] G_{1}(m)+ \\
& \quad+\left[\sum_{k_{1}=0}^{2} \Phi\left(k_{1}\right) \sum_{k_{2}=0}^{2} G_{2.2}\left(k_{2}\right)\left[C\left(k_{1}+k_{2}\right)-C\left(k_{1}+k_{2}+2\right)\right]\right] G_{1.1}(m) \\
& =F(m), \quad m=1,2,3
\end{aligned}
$$

Which is equivalent to the system

$$
\left.\begin{array}{l}
-\frac{7 \pi}{8} \Phi(0)+\frac{9 \pi}{16} \Phi(2)=1, \\
-\pi \Phi(1)=1 \\
\frac{\pi}{2} \Phi(0)-\frac{7 \pi}{8} \Phi(2)=1 .
\end{array}\right\}
$$

Solving the system (38) yields

$$
\left.\Phi(0)=\frac{-92}{31 \pi}, \quad \Phi(1)=0, \quad \Phi(2)=\frac{-88}{31 \pi} .\right\}
$$

Thus the approximate solution of Eq.(33) is

$$
g(x)=\sqrt{1-x^{2}}\left(\frac{-92}{31 \pi}-\frac{88}{31 \pi} x^{2}\right) .
$$

which is identical to the exact solution. 


\begin{tabular}{|c|c|c|}
\hline \multirow[b]{2}{*}{$x$} & \multicolumn{2}{|c|}{ Errors } \\
\hline & Method in [10] $(N=20)$ & Proposed method $(N=2)$ \\
\hline$-9.500000 \mathrm{E}-01$ & $0.000000 \mathrm{E}+00$ & $0.000000 \mathrm{E}+00$ \\
\hline$-9.000000 \mathrm{E}-01$ & $5.960464 \mathrm{E}-08$ & $0.000000 \mathrm{E}+00$ \\
\hline$-7.000000 \mathrm{E}-01$ & 1.192093E-07 & $0.000000 \mathrm{E}+00$ \\
\hline$-5.000000 \mathrm{E}-01$ & 1.192093E-07 & $0.000000 \mathrm{E}+00$ \\
\hline$-3.000000 \mathrm{E}-01$ & $1.788139 \mathrm{E}-07$ & $0.000000 \mathrm{E}+00$ \\
\hline$-1.000000 \mathrm{E}-01$ & $1.788139 \mathrm{E}-07$ & $0.000000 \mathrm{E}+00$ \\
\hline $0.000000 \mathrm{E}+00$ & $1.788139 \mathrm{E}-07$ & $0.000000 \mathrm{E}+00$ \\
\hline $1.000000 \mathrm{E}-01$ & $1.788139 \mathrm{E}-07$ & $0.000000 \mathrm{E}+00$ \\
\hline $3.000000 \mathrm{E}-01$ & $1.788139 \mathrm{E}-07$ & $0.000000 \mathrm{E}+00$ \\
\hline $5.000000 \mathrm{E}-01$ & 1.192093E-07 & $0.000000 \mathrm{E}+00$ \\
\hline $7.000000 \mathrm{E}-01$ & 1.192093E-07 & $0.000000 \mathrm{E}+00$ \\
\hline $9.000000 \mathrm{E}-01$ & $5.960464 \mathrm{E}-08$ & $0.000000 \mathrm{E}+00$ \\
\hline $9.500000 \mathrm{E}-01$ & $0.000000 \mathrm{E}+00$ & $0.000000 \mathrm{E}+00$ \\
\hline
\end{tabular}

Example 3. Consider the following integral equation

$$
\int_{-1}^{1} \frac{g(t)}{t-x} d t+\int_{-1}^{1}\left(x^{2}+t^{2}\right) g(t) d t=\frac{-3}{2} x^{2}+2 x, \quad-1<x<1
$$

It is not difficult to verify that the exact solution of Eq.(40) is

$$
g(x)=\sqrt{1-x^{2}}\left(\frac{-2}{\pi}+\frac{1}{2 \pi} x\right)
$$

It is clear that the differential Transform of $f(x)=\frac{-3}{2} x^{2}+2 x$ is

$$
\left.\begin{array}{l}
F(0)=0, \\
F(1)=2, \\
F(2)=\frac{-3}{2}, \\
F(k)=0, k=3,4, \ldots
\end{array}\right\}
$$


It is clear that $k(x, t)=x^{2}+t^{2}$, which is separable kernel.

Let $g_{1}(x)=x^{2}, g_{2}(x)=1, g_{1.1}(x)=1, g_{2.2}(x)=t^{2}$, then the differential transform of $g_{1}, g_{2}, g_{1.1}$ and $g_{2.2}$ respectively are

$$
\left.\begin{array}{l}
G_{1}(0)=0, G_{1}(1)=0, G_{1}(2)=1, G_{1}(3)=0, \\
G_{2}(0)=1, G_{2}(1)=0, G_{2}(2)=0, G_{2}(3)=0, \\
G_{1.1}(0)=1, G_{1.1}(1)=0, G_{1.1}(2)=0, G_{1.1}(3)=0, \\
G_{2.2}(0)=0, G_{2.2}(1)=0, G_{2.2}(2)=1, G_{2.2}(3)=0 .
\end{array}\right\}
$$

Using the system (24) with $N=2$, we have

$$
\begin{aligned}
& \sum_{k=1}^{2} \Phi(k) \sum_{k_{1}=0}^{k-1} \delta\left(m-k_{1}\right)\left[C\left(k-k_{1}-1\right)-C\left(k-k_{1}+1\right)\right]- \\
& \quad-\pi \sum_{k_{2}=0}^{m} \delta\left(k_{2}-1\right) \Phi\left(m-k_{2}\right)+ \\
& \quad+\left[\sum_{k_{1}=0}^{2} \Phi\left(k_{1}\right) \sum_{k_{2}=0}^{2} G_{2}\left(k_{2}\right)\left[C\left(k_{1}+k_{2}\right)-C\left(k_{1}+k_{2}+2\right)\right]\right] G_{1}(m)+ \\
& \quad+\left[\sum_{k_{1}=0}^{2} \Phi\left(k_{1}\right) \sum_{k_{2}=0}^{2} G_{2.2}\left(k_{2}\right)\left[C\left(k_{1}+k_{2}\right)-C\left(k_{1}+k_{2}+2\right)\right]\right] G_{1.1}(m) \\
& =F(m), \quad m=1,2,3
\end{aligned}
$$

Which is equivalent to the system

$$
\left.\begin{array}{l}
-\pi \Phi(0)+\frac{\pi}{2} \Phi(2)=2, \\
\frac{\pi}{2} \Phi(0)-\pi \Phi(1)+\frac{\pi}{8} \Phi(2)=\frac{-3}{2} \\
-\pi \Phi(2)=0
\end{array}\right\}
$$

Solving the system (45) yields

$$
\left.\Phi(0)=\frac{-2}{\pi}, \quad \Phi(1)=\frac{1}{2 \pi}, \quad \Phi(2)=0 .\right\}
$$

Thus the approximate solution of Eq.(40) is

$$
g(x)=\sqrt{1-x^{2}}\left(\frac{-2}{\pi}+\frac{1}{2 \pi} x\right) .
$$

which is identical to the exact solution. 


\begin{tabular}{|c|c|c|}
\hline \multirow[b]{2}{*}{$x$} & \multicolumn{2}{|c|}{ Errors } \\
\hline & Method in[10] $(N=20)$ & Proposed method ( $N=2)$ \\
\hline$-9.500000 \mathrm{E}-01$ & 2.980232 E-08 & $0.000000 \mathrm{E}+00$ \\
\hline$-9.000000 \mathrm{E}-01$ & 2.980232 E-08 & $0.000000 \mathrm{E}+00$ \\
\hline$-7.000000 \mathrm{E}-01$ & $0.000000 \mathrm{E}+00$ & $0.000000 \mathrm{E}+00$ \\
\hline$-5.000000 \mathrm{E}-01$ & $0.000000 \mathrm{E}+00$ & $0.000000 \mathrm{E}+00$ \\
\hline$-3.000000 \mathrm{E}-01$ & $0.000000 \mathrm{E}+00$ & $0.000000 \mathrm{E}+00$ \\
\hline$-1.000000 \mathrm{E}-01$ & $5.960464 \mathrm{E}-08$ & $0.000000 \mathrm{E}+00$ \\
\hline $0.000000 \mathrm{E}+00$ & 5.960464E-08 & $0.000000 \mathrm{E}+00$ \\
\hline $1.000000 \mathrm{E}-01$ & 5.960464E-08 & $0.000000 \mathrm{E}+00$ \\
\hline $3.000000 \mathrm{E}-01$ & 1.192093E-07 & $0.000000 \mathrm{E}+00$ \\
\hline $5.000000 \mathrm{E}-01$ & 1.192093E-07 & $0.000000 \mathrm{E}+00$ \\
\hline $7.000000 \mathrm{E}-01$ & 1.192093E-07 & $0.000000 \mathrm{E}+00$ \\
\hline $9.000000 \mathrm{E}-01$ & 8.940697 E-08 & $0.000000 \mathrm{E}+00$ \\
\hline $9.500000 \mathrm{E}-01$ & $5.960464 \mathrm{E}-08$ & $0.000000 \mathrm{E}+00$ \\
\hline
\end{tabular}

Example 4. Consider the following integral equation

$$
\int_{-1}^{1} \frac{g(t)}{t-x} d t+\int_{-1}^{1}(t-x)^{2} g(t) d t=-\pi\left(4 x^{3}-3 x-\frac{1}{8}\right), \quad-1<x<1
$$

It is not difficult to verify that the exact solution of Eq.(47) is

$$
g(t)=\sqrt{1-t^{2}}\left(4 t^{2}-1\right)
$$

It is clear that the differential Transform of $f(x)=-4 \pi x^{3}+3 \pi x+\frac{\pi}{8}$ is

$$
\left.\begin{array}{l}
F(1)=3 \pi, \\
F(2)=0, \\
F(3)=-4 \pi, \\
F(k)=0, k=4,5, \ldots
\end{array}\right\}
$$

It is clear that $k(x, t)=(t-x)^{2}$, which is Convolution Kernel. 
Let $f_{1}(x-t)=(t-x)^{2}$, then the differential transform of $g$ is

$$
\left.F_{1}(1)=0, \quad F_{1}(2)=1, \quad F_{1}(3)=0 .\right\}
$$

Using the system (25) with $N=2$, we have

$$
\begin{aligned}
& \sum_{k=1}^{2} \Phi(k) \sum_{k_{1}=0}^{k-1} \delta\left(m-k_{1}\right)\left[C\left(k-k_{1}-1\right)-C\left(k-k_{1}+1\right)\right]- \\
& \quad-\pi \sum_{k_{2}=0}^{m} \delta\left(k_{2}-1\right) \Phi\left(m-k_{2}\right) \\
& +\sum_{k_{1}=0}^{2} \Phi\left(k_{1}\right) \sum_{k_{2}=0}^{2} F\left(k_{2}\right) \sum_{k=0}^{k_{2}}(-1)^{k}\left(\begin{array}{l}
k_{2} \\
k
\end{array}\right) \delta\left(k_{2}-k-m\right)\left(C\left(k+k_{1}\right)-C\left(k+k_{1}+2\right)\right) \\
& =F(m), \quad m=1,2,3
\end{aligned}
$$

Which is equivalent to the system

$$
\left.\begin{array}{l}
\Phi(0)+\frac{1}{4} \Phi(1)-\frac{1}{2} \Phi(2)=-3 \\
\frac{1}{2} \Phi(0)-\Phi(1)+\frac{1}{8} \Phi(2)=0 \\
\Phi(2)=4
\end{array}\right\}
$$

Solving the system (52) yields

$$
\Phi(0)=-1, \quad \Phi(1)=0, \quad \Phi(2)=4 .\}
$$

Thus the approximate solution of Eq.(47) is

$$
g(x)=\sqrt{1-x^{2}}\left(-1+4 x^{2}\right) .
$$

which is identical to the exact solution. 


\section{REFERENCES}

1. Abdulkawi, M., A Numerical Solution of Singular Integral Equations. International Journal of Mathematics and Statistics, Volume 10, Number W11(2011), 69-76.

2. M. Abdulkawi and M. Y. H. Alyami (2015). Bounded solution of Characteristic singular integral equation using differential transform method. Proceedings of the 7th International Conference on Research and Education in Mathematics (ICREM7), 25-27. DOI:10.1109/ICREM.2015.7357024.

3. Amit, Setia., Numerical solution of various cases of Cauchy type singular integral equation. Applied Mathematics and Computation, 230 (2014), 200-207.

4. Aytac, Arikoglu and Ibrahim, Ozkol., Solutions of integral and integro-differential equation systems by using differential transform method. Computers and Mathematics with Applications, 56 (2008), 24112417.

5. A. Dezhborda, Taher Lotfia and Katayoun, Mahdiania., A new efficient method for a case of the singular integral equation of the first kind. Journal of Computational and Applied Mathematics, 15 (2014), $0377-0427$.

6. Chakrabarti, A. and Berghe, V.G., Approximate Solution of Singular Integral Equations. Appl. Math. Lett. 17 (2004), 553-559.

7. Eshkuvatov, Z.K., Nik Long, N. M. A. and Abdulkawi, M., Approximate solution of singular integral equations of the first kind with Cauchy kernel. Appl. Math. Lett. 22 (2009), 651-657.

8. Eshkuvatov, Z.K., Nik Long, N. M. A. and Abdulkawi, M., On the Semi-bounded Solution of Cauchy Type Singular Integral Equations of the First Kind. European Journal of Pure and Applied Mathematics Vol. 2, No. 3 (2009), 462-472.

9. Mohamed S. Akel and Hussein S. Hussein., Numerical treatment of solving singular integral equations by using Sinc approximations. Applied Mathematics and Computation lett. 218 (2011), 3565-3573.

10. Samah, M. Dardery and Mohamed, M. Allan., Polynomials for Solving a Class of Singular Integral Equations. Applied Mathematics lett. 5 (2014), 753-764.

11. Lifanov, I.K. (1996). singular integral equations and discrete vortices. VSP BV, the Netherlands.

12. Kanwal, R. P. (1997). Linear Integral Equations, Theory and Technique. Second Edition. Birkhauser. Boston. 